\title{
Finite Coverings by Translates of Centrally Symmetric Convex Domains
}

\author{
G. Fejes Tóth \\ Mathematical Institute of the Hungarian Academy of Sciences, Reáltonada 13-15, \\ 1053 Budapest, Hungary
}

\begin{abstract}
Bambah and Rogers proved that the area of a convex domain in the plane which can be covered by $n$ translates of a given centrally symmetric convex domain $C$ is at most $(n-1) h(C)+a(C)$, where $h(C)$ denotes the area of the largest hexagon contained in $C$ and $a(C)$ stands for the area of $C$. An improvement with a term of magnitude $\sqrt{n}$ is given here. Our estimate implies that if $C$ is not a parallelogram, then any covering of any convex domain by at least 26 translates of $C$ is less economic than the thinnest covering of the whole plane by translates of $C$.
\end{abstract}

\section{Introduction}

By a domain we mean a bounded closed set in the plane with interior points. How large can the area $a(K)$ of a convex domain $K$, which can be covered by $n$ translates of a given centrally symmetric convex domain $C$ be? Let $h(C)$ denote the maximum area of a hexagon contained in $C$. We recall a theorem of Bambah and Rogers [1] which claims that

$$
a(K) \leq(n-1) h(C)+a(C)
$$

In the case when $C$ is a circle and $n \geq 2$ a stronger inequality holds [4]:

$$
a(K)<n h(C)
$$

These estimates give the asymptotically right order of magnitude of the maximum area of a convex domain which can be covered by $n$ translates of $C$. However, we shall show that they can be improved by a remainder term of magnitude $\sqrt{n}$. 
Let $q(C)$ denote the maximum area of a quadrangle contained in $C$. Then the following theorem holds:

Theorem. If a convex domain $K$ is covered by $n \geq 2$ translates of a centrally symmetric convex domain $C$ then

$$
\begin{aligned}
a(K) \leq & (n-1) h(C)+a(C) \\
& -\frac{h(C)-q(C)}{2(a(C)+q(C))} \\
& \times\left\{\sqrt{(a(C)+h(C))^{2}+(a(C)+q(C))[4(n-1) h(C)+3 a(C)-q(C)]}\right. \\
& -a(C)-h(C)\} .
\end{aligned}
$$

It is known [5], [7] that the least density $\theta(C)$ of a covering of the plane by translates of $C$ is equal to $a(C) / h(C)$. The finite covering density

$$
\theta_{n}(C)=\inf \left\{\frac{n a(C)}{a(K)}, K \text { can be covered by } n \text { translates of } C\right\}
$$

was introduced in [6]. In the case when $C$ is a parallelogram we obviously have $\theta_{n}(C)=\theta(C)=1$ for all $n$. In the following we consider the case when $C$ is not a parallelogram. Now our theorem implies that $\theta_{n}(C)>\theta(C)$ if $n$ is sufficiently large. Moreover, an easy calculation shows that the right-hand side of $(1)$ is less than $n h(C)$, hence $\theta_{n}(C)>\theta(C)$, if

$$
\begin{aligned}
n> & \frac{(a(C)+q(C))(a(C)-h(C))^{2}}{h(C)(h(C)-q(C))^{2}}+\frac{(a(C)-h(C))(a(C)+h(C))}{h(C)(h(C)-q(C))} \\
& -\frac{3 a(C)-q(C)}{4 h(C)}+1 .
\end{aligned}
$$

According to a theorem of Dowker [3] there is a parallelogram $P$ of area $q(C)$ inscribed into $C$, the centre of symmetry of which coincides with that of $C$. We observe that to any side $x y$ of $P$ which is not an arc of bd $C$ there is a triangle $x y z$ contained in the segment of $C$ cut off by $x y$ with an area at least half of the respective segment. It immediately follows that

$$
a(C)-h(C)<\frac{3}{4}(a(C)-q(C)) .
$$

Therefore

$$
\begin{gathered}
\frac{a(C)-h(C)}{h(C)-q(C)}<3, \\
\frac{3 a(C)-q(C)}{h(C)}>\frac{10 a(C)}{3 h(C)}-\frac{4}{3} \geq 2,
\end{gathered}
$$

and

$$
\frac{a(C)+q(C)}{h(C)}<4 \frac{a(C)+q(C)}{a(C)+3 q(C)}
$$


A theorem of Sas [8] states that

$$
\frac{q(C)}{a(C)} \geq \frac{2}{\pi} \text { and } \frac{h(C)}{a(C)} \geq \frac{\sqrt{27}}{2 \pi}
$$

From this, and the fact that the function $(1+x) /(1+3 x)$ is decreasing, it follows that

$$
\frac{a(C)+q(C)}{h(C)} \leq 4 \frac{\pi+2}{\pi+6}<2.25
$$

and

$$
\frac{a(C)+h(C)}{h(C)} \leq 1+\frac{2 \pi}{\sqrt{27}}<2.21
$$

The inequalities (3), (4), (5), and (6) imply that the right-hand side of (2) is less than 26 for all centrally symmetric convex domains $C$ other than a parallelogram. Thus we have the following:

Corollary. We have for all centrally symmetric convex domains $C$ other than $a$ parallelogram and all integers $n \geq 26$

$$
\theta_{n}(C)>\theta(C)
$$

It may be conjectured that this inequality holds for $n \geq 2$. The result in [3] quoted above confirms this conjecture in the case when $C$ is a circle.

Our theorem implies that the area of a convex domain which is covered by $n$ unit circles is at most

$$
\frac{3 \sqrt{3}}{2} n-0.425 \sqrt{n}+0.878
$$

This may be compared with a result of Gritzmann and Wills [6] according to which there is a convex domain of area

$$
\frac{3 \sqrt{3}}{2} n-2.755 \sqrt{n}
$$

which can be covered by $n$ unit circles.

The proof of the Theorem is given in Section 4. The main tool to the proof is a special formula for the area of a convex domain covered by translates of $C$ discussed in Section 3. This, in turn, is based on the construction of generalized Dirichlet cells described in Section 2. 


\section{Generalized Dirichlet Cells}

We use an obvious vector notation, so that a point of the plane and the vector pointing from the origin $o$ to the respective point will be identified. For a real number $\lambda$, a point $p$ and a point set $A, \lambda A+p$ will denote the set of points $\lambda x+p$ with $x$ in $A$. The boundary of a set $A$ will be denoted by bd $A$. Throughout the paper we assume that

(i) $C$ is a strictly convex domain with centre of symmetry at the origin o.

We consider the distance function $f(x)$ of $C$ defined by

$$
f(x)=\inf _{\substack{\lambda>0 \\ x \in \lambda C}} \lambda
$$

The function $f(x)$ is continuous for all $x$ and it satisfies

$$
f(x+y) \leq f(x)+f(y)
$$

for all points $x$ and $y$, with strict inequality unless $x=\lambda y$ for some $\lambda \geq 0$ or $y=0$. Further, we have

$$
f(\lambda x)=|\lambda| f(x)
$$

for all points $x$ and all real numbers $\lambda$ (see e.g. [9]). The sets $\lambda C+a$ and $\operatorname{bd}(\lambda C+a)$ consist of the points $x$ with $f(x-a) \leq|\lambda|$ and $f(x-a)=|\lambda|$, respectively.

We reformulate Lemma 1 from [2] as:

Proposition 1. For any two distinct points $p$ and $q$ and any real numbers $\lambda$ and $\mu$ $\mathrm{bd}(\lambda C+p)$ and $\mathrm{bd}(\mu C+q)$ have at most two points in common.

Hence it immediately follows:

Proposition 2. If $x, y$, and $z$ are distinct points then there is at most one point $p$ for which $f(x-p)=f(y-p)=f(z-p)$.

For, if we had $f(x-p)=f(y-p)=f(z-p)=\lambda$ and $f(x-q)=f(y-q)=$ $f(z-q)=\mu$ for distinct points $p$ and $q$, then $x, y$, and $z$ would be three points belonging to $(\mathrm{bd}(\lambda C+p)) \cap(\mathrm{bd}(\mu C+q))$, in contradiction to Proposition 1 .

Let $p$ and $q$ be distinct points and let $x$ move on a half-ray with endpoint $p$. It follows by (7) that the quantity $f(x-p)-f(x-q)$ is then a strictly increasing function of the distance of $x$ to $p$. Together with the continuity of $f(x)$ this implies:

Proposition 3. For any two distinct points $p$ and $q$ the set of points $x$ with $f(x-p)=f(x-q)$ is a continuous curve. It separates the sets of points $x$ with $f(x-p)<f(x-q)$ and $f(x-p)>f(x-q)$, which are sta: shaped with centres $p$ and $q$, respectively. 
Suppose now that $p$ and $q(p \neq q)$ are contained in the convex domain $K$. Then the midpoint $m$ of the segment $p q$ is contained in $K$, and we have $f(m-p)=$ $f(m-q)$. We recall Lemma 6 from [2]. It states that the set of points $x$ with $f(x-p)=f(x-q)$ has points on both sides of the line $p q$ arbitrarily far from this line. It follows that there is a point of intersection of bd $K$ with the curve consisting of the points $x$ with $f(x-p)=f(x-q)$ on both sides of the line $p q$. Suppose that there are two points on bd $K$, say $x$ and $y$, on the same side of the line $p q$ such that $f(x-p)=f(x-q)$ and $f(y-p)=f(y-q)$. Then either the open segments $p x$ and $q y$ or the open segments $p y$ and $q y$ intersect in a point, say $z$. Proposition 3 implies in any case that for the point $z$ we have $f(z-p)<f(z-q)$ and simultaneously $f(z-p)>f(z-q)$. Since this is impossible, we obtain:

Proposition 4. If $p$ and $q$ are distinct points in the convex domain $K$, then there are exactly two points $x$ and $y$ on bd $K$ such that $f(x-p)=f(x-q)$ and $f(y-p)=$ $f(y-q)$.

As above, let $K$ be a convex domain and $a_{1}, \ldots, a_{n}$ distinct points such that

(ii) $a_{i} \in K$ for $i=1, \ldots, n$.

Let $D_{i}$ consist of those points $x$ of $K$ for which $f\left(x-a_{i}\right) \leq f\left(x-a_{j}\right)$ for all $j=1, \ldots, n . D_{i}$ will be called a generalized Dirichlet cell associated with $a_{i}$. It follows by Proposition 3 and assumption (ii) that the sets $D_{i}, i=1, \ldots, n$, are closed star-shaped sets which cover $K$, up to common boundary points exactly once. Let $D_{0}$ be the closure of the complement of $K$. The sets $D_{i}, i=0, \ldots, n$, constitute the faces of a cell complex $\mathscr{C}$. A point of the plane is said to be a vertex of $\mathscr{C}$ if it belongs to the boundary of at least three faces of $\mathscr{C}$. An edge of $\mathscr{C}$ is a connected component of the intersection of the boundaries of two faces of $\mathscr{C}$ containing more than one point.

Proposition 5. Suppose that

(iii) for any point $x$ in the plane and any real number $\lambda$, bd $(\lambda C+x)$ contains at most three of the points $a_{i}, i=1, \ldots, n$, and, moreover, if $x \in$ bd $K$, then $\operatorname{bd}(\lambda C+x)$ contains at most two of these points.

Then each vertex of the cell complex $\mathscr{C}$ is trihedral and $\mathscr{C}$ has exactly $2 n-2$ vertices.

The first statement of Proposition 5 is obvious. Using the fact that the cell complex $\mathscr{C}$ is trihedral, the second statement follows easily by Euler's formula.

\section{Two Lemmas Concerning Covering Convex Domains by Translates of $C$}

In this section we use the notations of the previous one. We assume that the sets $C, K$ and $\left\{a_{i}\right\}_{i=1}^{n}$ satisfy conditions (i)-(iii). We write $C_{i}=C+a_{i}$ and assume that

(iv) $K \subset \bigcup_{i=1}^{n} C_{i}$, but no proper subset of $\left\{C_{i}\right\}_{i=1}^{n}$ covers $K$. 
We consider the cell complex $\mathscr{C}$ introduced in Section 2 , and observe that now we have, for $i=1, \ldots, n, D_{i} \subset C_{i}$. For, if we had a point $x$ which is in $D_{i}$ but not in $C_{i}$, then we would have $f\left(x-a_{j}\right)>1$ for all $j=1, \ldots, n$. But then $x$ would not be covered by the sets $C_{i}, i=1, \ldots, n$.

Let $E$ and $V$ denote the set of edges and vertices of $\mathscr{C}$, respectively. With each edge $e \in E$ we associate a set $L(e)$ as follows: if $e$ is an edge common to the cells $D_{i}$ and $D_{j}$, and $i, j \neq 0$, then let $L(e)=C_{i} \cap C_{j}$. If, on the other hand, $e$ is an edge common to $D_{i}$ and $D_{0}$, then let $L(e)$ be the connected component of $C_{i} \cap D_{0}$, the boundary of which contains $e$. For a vertex $v \in V$, in which the edges $e_{1}, e_{2}$, and $e_{3}$ meet, let $I(v)$ be the connected component of $L\left(e_{1}\right) \cap L\left(e_{2}\right) \cap L\left(e_{3}\right)$ which contains $v$. We write

$$
t(v)=a\left(L\left(e_{1}\right)\right)+a\left(L\left(e_{2}\right)\right)+a\left(L\left(e_{3}\right)\right)-2 a(I(v)) .
$$

Now we are in the position to give the formula for $a(K)$ announced in the Introduction:

Lemma 1. We have

$$
a(K)=n a(C)-\frac{1}{2} \sum_{v \in V} t(v) .
$$

This formula has been proved in [4] for the case when $C$ is a circle. However, the proof given in [4] is too concise and needs a minor correction. In the following we give a detailed proof in the general case.

We consider a set $C_{i}=C+a_{i}$ and the generalized Dirichlet cell $D_{i}$ associated with $a_{i}$. The edges of $\mathscr{C}$ bounding $D_{i}$ will be called the sides of $D_{i}$. Let $v_{1}, \ldots, v_{r}$ be the vertices of $\mathscr{C}$ on bd $D_{i}$ enumerated in their natural cyclic order when going on bd $D_{i}$ in the clockwise direction. We write $v_{t+1}=v_{1}$. The side of $D_{i}$ joining $v_{m}$ to $v_{m+1}(m=1, \ldots, r)$ will be denoted by $s_{m}$. We associate with $s_{m}$ an arc $s_{m}^{*}$, called the chord of $C_{i}$, determined by $s_{m}$ as follows: if $s_{m}$ is an $\operatorname{arc}$ of bd $K$ then let $s_{m}^{*}$ be the connected component of $C_{i} \cap$ bd $K$ containing $s_{m}$. If, on the other hand, $D_{i}$ abuts along $s_{m}$ on a cell $D_{h}$ of $\mathscr{C}$ other than $D_{0}$, then $s_{m}^{*}$ is defined as the set of points $x$ in $C_{i}$ for which $f\left(x-a_{i}\right)=f\left(x-a_{h}\right)$. The fact that the set $s_{m}^{*}$ is a Jordan arc is obvious in the first case, and it follows immediately also in the second case by Propositions 3 and 4. Moreover, $D_{i} \subset C_{i}$ implies that $s_{m} \subset s_{m}^{*}$. We observe that in the case when $s_{m}$ is an edge of $\mathscr{C}$ common to $D_{i}$ and $D_{h} \neq D_{0}$, $s_{m}^{*}$ is contained in $C_{i} \cap C_{h}$ and its endpoints are just the points of intersection of bd $C_{i}$ and bd $C_{h}$. The chord $s_{m}^{*}$ cuts the "segment" $S_{m}$ off from $C_{i} . S_{m}$ consists of the points $x$ of $C_{i}$ for which $f\left(x-a_{i}\right) \geq f\left(x-a_{h}\right)$, if, along $s_{m}$, the cell $D_{h} \neq D_{0}$ joins to $D_{i}$, and $S_{m}=L\left(s_{m}\right)$ otherwise.

Let $p_{m}$ and $q_{m}$ be the endpoints of $s_{m}^{*}$, and suppose that the notations are chosen so that traveling on $s_{m}^{*}$ from $p_{m}$ to $q_{m}$ we first meet $v_{m}$ and then $v_{m+1}$. Let $s_{m}^{+}$and $s_{m}^{-}$be the open arcs of $s_{m}^{*}$ from $v_{m}$ through $v_{m+1}$ to $q_{m}$ and from $p_{m}$ through $v_{m}$ to $v_{m+1}$, respectively. Then we have:

Proposition 6. For every pair $j, k(1 \leq j \neq k \leq n)$, the arcs $s_{j}^{-}$and $s_{k}^{+}$, as well as the $\operatorname{arcs} s_{j}^{+}$and $s_{\bar{k}}^{-}$, intersect at, at most, one point. Furthermore, we have

$$
s_{j}^{-} \cap s_{k}^{-}=\varnothing \quad \text { and } s_{j}^{+} \cap s_{k}^{+}=\varnothing \text {. }
$$


Consider first the case when $s_{j}^{*}$ and $s_{k}^{*}$ intersect at a single point, say $x$. Then we have to verify only the relations (8). Now the cyclic order of the points $p_{j}$, $p_{k}, q_{j}$, and $q_{k}$ on bd $C_{i}$ is of the type $p, p, q, q$. Without loss of generality we assume that we meet these points in the order $p_{j}, p_{k}, q_{j}$, and $q_{k}$ when going on bd $C_{i}$ in the clockwise direction. Then $v_{j}$ and $v_{j+1}$ are on the arc of $s_{j}^{*}$ between $p_{j}$ and $x$, while $v_{k}$ and $v_{k+1}$ are on the arc of $s_{k}^{*}$ between $x$ and $q_{k}$. This implies (8).

Now suppose that $s_{j}^{*}$ and $s_{k}^{*}$ have more than one point in common. By Proposition 2 then at least one of $s_{j}$ and $s_{k}$, say $s_{j}$, is an $\operatorname{arc}$ of bd $K$. If $s_{k}$ abuts on a cell $D_{h}$ other than $D_{0}$, then $s_{j}$ and $s_{k}$ intersect at exactly two points by Propositon 4. Moreover, one of the points of intersection, say $x$, lies on the closed arc of $s_{k}^{*}$ from $p_{k}$ to $v_{k}$, and the other one, say $y$, lies between $v_{k+1}$ and $q_{k}$. If $v_{j}$ and $v_{j+1}$ are situated on $s_{j}^{*}$ between $x$ and $y$, then the order of points on $s_{j}^{*}$ is $p_{j}, y, v_{j}, v_{j+1}, x$, and $q_{j}$, and it is easy to check that the statements of Proposition 6 are true. Otherwise the subset of those points $z$ of $K$ for which $f\left(z-a_{i}\right) \geq$ $f\left(z-a_{h}\right)$ is enclosed by the arcs of $s_{j}^{*}$ and $s_{k}^{*}$ between $x$ and $y$. Therefore this subset of $K$ is contained in $S_{k}$, and consequently also in $C_{i}$. On the other hand, $D_{h}$ is contained in this subset of $K$, so that we have $D_{h} \subset C_{i}$. But this means that $C_{h}$ can be omitted from the sets $C_{i}, i=1, \ldots, n$, and we still obtain a covering of $K$, which is impossible by assumption (iv).

It remains to consider the case when, as well as $s_{j}, s_{k}$ is also an arc of bd $K$. Then the supposition that $s_{j}^{*} \cap s_{k}^{*}$ contains more than one point implies that $s_{j}^{*}$ and $s_{k}^{*}$ are identical. Since, however, $s_{j} \neq s_{k}$, there is a side of $D_{i}$ between $s_{j}$ and $s_{k}$, say $s_{l}$, such that $D_{i}$ joins along $s_{l}$ to a cell of $\mathscr{C}$ other than $D_{0}$. Moreover, $s_{l}^{*}$ intersects $s_{j}^{*}$ in two points, say $x$ and $y$, so that $v_{j}$ and $v_{j+1}$ are not contained in the arc of $s_{j}^{*}$ between $x$ and $y$. However, this is just the situation from which we have seen above that it contradicts assumption (iv). This completes the proof of Proposition 6.

We have

$$
a\left(D_{i}\right)=a(C)-a\left(\bigcup_{m=1}^{r} S_{m}\right)
$$

We write $R=\{1, \ldots, r\}$. Then a well-known formula states that

$$
a\left(\bigcup_{m=1}^{r} S_{m}\right)=\sum_{m=1}^{r}(-1)^{m-1} \sum_{\substack{M \subset R \\|M|=m}} a\left(\bigcap_{j \in M} S_{j}\right) .
$$

Here $|M|$ denotes the cardinality of $M$.

Let us suppose that for some $j$ and $k, S_{j} \cap S_{k} \neq \varnothing$. Then Proposition 6 implies that $S_{j} \cap S_{k}$ is either connected or it is composed of two connected components, one containing the point $s_{j}^{+} \cap s_{k}^{-}$and the other containing $s_{j}^{-} \cap s_{k}^{+}$. Let $S$ be a connected component of $S_{j} \cap S_{k}$. Without loss of generality we suppose that $x=s_{j}^{+} \cap s_{k}^{-} \in S$. Let $s_{l}$ be a side of $D_{i}$ which lies between $s_{j}$ and $s_{k}$ when we go around bd $D_{i}$ in the clockwise direction. Again, Proposition 6 implies that $p_{l}$ lies between $p_{j}$ and $p_{k}$, and $q_{i}$ lies between $q_{j}$ and $q_{k}$ when going on bd $C_{i}$ in the 
clockwise direction. Furthermore, when we go from $p_{l}$ to $q_{l}$ on $s_{l}^{*}$ we meet $s_{j}^{*}$ in a point between $p_{j}$ and $x$, and then we meet $s_{k}^{*}$ in a point between $x$ and $q_{k}$. This imples that

$$
S \subset S_{l}
$$

If there are exactly $t(0 \leq t \leq r-2)$ sides of $D_{i}$ between $s_{j}$ and $s_{k}$, then there are $\left(\begin{array}{c}t \\ m-2\end{array}\right)$ possibilities of obtaining $S$ as a connected component of the intersection of $m$ segments of $C_{i}$. This yields a contribution to the sum on the right-hand side of (10) equal to

$$
a(S) \sum_{m=1}^{t+2}(-1)^{m-1}\left(\begin{array}{c}
t \\
m-2
\end{array}\right)
$$

This sum is equal to $-a(S)$ if $t=0$, i.e., if $s_{j}$ and $s_{k}$ are adjacent sides of $D_{i}$, and it is equal to 0 otherwise. Thus, writing $S_{0}=S_{r}$ and denoting by $J_{m}$ the connected component of $S_{m-1} \cap S_{m}$ containing the vertex $v_{m}(m=1, \ldots, r)$, we have

$$
a\left(\bigcup_{m=1}^{r} S_{m}\right)=\sum_{m=1}^{r}\left[a\left(S_{m}\right)-a\left(J_{m}\right)\right]
$$

Hence we obtain by (9)

$$
a\left(D_{i}\right)=a(C)-\sum_{m=1}^{r}\left[a\left(S_{m}\right)-a\left(J_{m}\right)\right]
$$

Summing up this equality for $i=1, \ldots, n$ we obtain

$$
a(K)=\sum_{i=1}^{n} a\left(D_{i}\right)=n a(C)-\sum_{e \in E} a(L(e))+\sum_{v \in V} a(I(v))
$$

Now Lemma 1 follows by observing that

$$
\sum_{e \in E} a(L(e))-\sum_{v \in V} a(I(v))=\frac{1}{2} \sum_{v \in V} t(V)
$$

Lemma 2. Let $b$ denote the number of vertices of $\mathscr{C}$ which are situated on bd $K$. Then we have

$$
a(K) \leq(n-1) h(C)+a(C)-\frac{b}{2}(h(C)-q(C)) .
$$




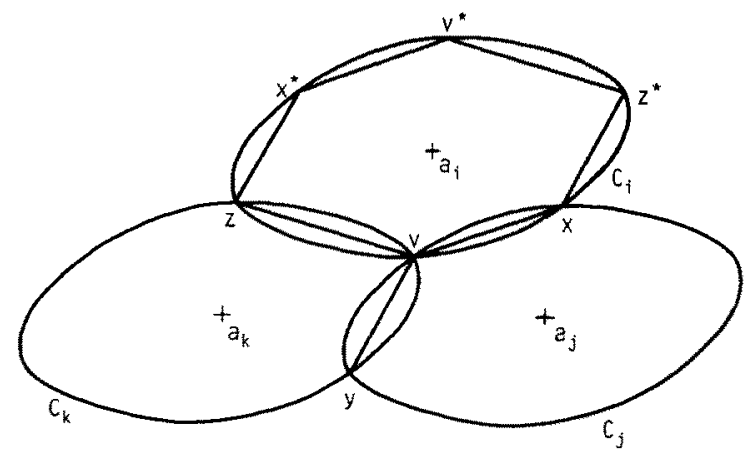

Fig. 1

Let $v$ be a vertex of $\mathscr{C}$ common to the cells $D_{i}, D_{j}$, and $D_{k}$, and assume that $i, j, k \neq 0$. Now $t(v)$ is nothing else but the area of the part of the plane which is covered at least twice by $C_{i}, C_{j}$, and $C_{k}$. Looking for a lower bound for $t(v)$, we may suppose that the intersection $C_{i} \cap C_{j} \cap C_{k}$ consists of the single point $v$. Then bd $C_{i}$ and bd $C_{j}$, bd $C_{j}$ and bd $C_{k}$, and bd $C_{k}$ and bd $C_{i}$ intersect at the points $x=a_{i}+a_{j}-v, y=a_{j}+a_{k}-v$, and $z=a_{k}+a_{i}-v$, respectively (Fig. 1). Let $x^{*}, z^{*}$, and $v^{*}$ be the points obtained from $x, z$, and $v$ by reflecting in $a_{i}$. Then we have $x^{*}=a_{i}-a_{j}+v, z^{*}=a_{i}-a_{k}+v$, and $v^{*}=2 a_{i}-v$. Now $v, x, z^{*}, v^{*}, x^{*}$, and $z$ are the vertices of a centrally symmetric convex hexagon $H$ inscribed into $C_{i}$. The sides $x z^{*}$ and $x^{*} z$ of $H$ are parallel to the segment $v y$. This implies that $t(v)=a(C)-a(H)$. Hence we obtain that

$$
t(v) \geq a(C)-h(C) \quad \text { if } \quad v \notin b d K .
$$

Now let $v$ be a vertex of 6 common to $D_{0}, D_{i}$, and $D_{j}$. Similarly as above, we assume that $v$ is the only point common to $D_{0}, D_{i}$, and $D_{j}$. Let $S$ be the closure of the complement of a supporting half-plane of $K$ such that $v \in$ bd $S$ (Fig. 2). Let $t^{*}(v)$ be the area of the part of the plane which is covered at least twice by the sets $C_{i}, C_{j}$, and $S$. Then we have $t(v) \geq t^{*}(v)$. Let the point of intersection of bd $S$ with bd $C_{i}$ other than $v$ be $p$, and that with bd $C_{j}$ be $q$. The area $t^{*}(v)$ will be minimal in the case when the segments $p v$ and $v q$ are of equal length. Otherwise we can decrease $t^{*}(v)$ by a small rotation of $S$ about $v$ in a suitable direction. Thus, let us suppose that $p v=v q$. Let the second point of intersection of bd $C_{i}$ and bd $C_{j}$ be $w$. Let the line through $w$ parallel to bd $S$ intersect bd $C_{i}$

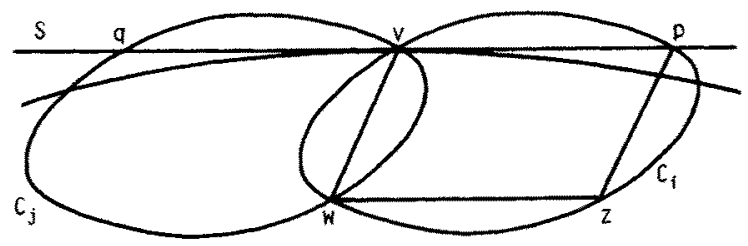

Fig. 2 
in another point $z$. Then $P=v w z p$ is a parallelogram inscribed into $C_{i}$, and we have $t^{*}(v)=a(C)-a(P)$. It follows that

$$
t(v) \geq a(C)-q(C) \quad \text { if } \quad v \in \text { bd } K .
$$

Lemma 2 is now an immediate consequence of Proposition 5, Lemma 1, and the relations (12) and (13).

\section{Proof of the Theorem}

First we show that it suffices to prove the theorem in the case when $C$ and its translates $C_{i}=C+a_{i}, i=1, \ldots, n$, covering $K$ satisfy conditions (i)-(iv).

Having proved the theorem in the case when $C$ is strictly convex, the general case can be settled by approximating $C$ by strictly convex domains containing it. It is also clear that the assumption in (iv), that no proper subset of $\left\{C_{i}\right\}_{i=1}^{n}$ covers $K$, does not affect the generality.

Suppose now that assumptions (i) and (iv) are satisfied, and for some $i$, $1 \leq i \leq n, a_{i} \notin K$. Then $K \cap C_{i} \neq \varnothing$. A standard continuity argument yields that there is a supporting line $l$ of $K$ such that $a_{i}$ and $K$ are separated by $l$ and the midpoint $m$ of the line segment $l \cap C_{i}$ is on bd $K$. Denoting the endpoints of $l \cap C_{i}$ by $p$ and $q$, we have $2 a_{i}-p \in$ bd $K$ and $2 a_{i}-q \in$ bd $K$. The set $C_{i} \cap K$ is then contained in the parallel strip bounded by the lines through $p$ and $2 a_{i}-q$ and $q$ and $2 a_{i}-p$, respectively. It follows that $C_{i} \cap K$ is contained in the set $C_{i}^{\prime}=C+m$. Thus $K$ is covered by the sets $C_{1}, \ldots, C_{i}^{\prime}, \ldots, C_{n}$. This justifies assumption (ii).

Finally, if (iii) is not satisfied then for any positive number $\varepsilon$ we can replace the set $\left\{a_{i}\right\}_{i=1}^{n}$ by another one satisfying (iii) so that $K \subset \bigcup_{i=1}^{n}\left((1+\varepsilon) C+a_{i}\right)$. This shows that (iii) can be assumed without loss of generality.

We observe that if $n=2$, then the cell complex $\mathscr{C}$ has two vertices both of them lying on bd $K$. Thus Lemma 2 implies that if $K$ is covered by two translates of $C$, then

$$
a(K) \leq a(C)+q(C) .
$$

An easy calculation shows that if for some positive numbers $\lambda$ and $\mu$ and some points $p$ and $q$ the intersection $(\lambda C+p) \cap(\mu C+q)$ is not empty, then

$$
(\lambda C+p) \cup(\mu C+q) \subset(\lambda+\mu) C+\frac{\lambda p+\mu q}{\lambda+\mu} .
$$

Let $e_{1}, \ldots, e_{b}$ be the edges of $\mathscr{C}$ which are arcs of bd $K$ enumerated in their natural cyclic order. Let $i_{j}$ be the index for which $e_{j} \subset D_{0} \cap D_{i},(j=1, \ldots, b)$. Then we have

$$
\text { bd } K \subset \bigcup_{j=1}^{b} C_{i_{j}} \text { and } \quad C_{i_{j}} \cap C_{i_{j+1}} \neq \varnothing \quad \text { for } \quad j=1, \ldots, b-1 \text {. }
$$


Writing $r=[(b+1) / 2]$ and $s=[(b+2) / 2]$, we obtain by induction that

$$
\text { bd } K \subset\left(r C+\frac{1}{r} \sum_{j=1}^{r} a_{i_{j}}\right) \cup\left(r C+\frac{1}{r} \sum_{j=s}^{b} a_{i_{j}}\right) \text {. }
$$

We note that if two convex domains cover bd $K$, then they cover $K$ as well. Thus, $K$ can be covered by two translates of $((b+1) / 2) C$. Hence we obtain by $(14)$ that

$$
a(K) \leq\left(\frac{b+1}{2}\right)^{2}(a(C)+q(C)) .
$$

It is easy to check that the right hand-sides of (11) and (15) are equal if

$$
\begin{aligned}
& b=b_{0}=\frac{1}{a(C)+q(C)} \\
& \times\left\{\sqrt{(a(C)+h(C))^{2}+(a(C)+q(C))[4(n-1) h(C)+3 a(C)-q(C)]}-a(C)-h(C)\right\} .
\end{aligned}
$$

For $b<b_{o}(15)$, for $b>b_{0}(11)$, is stronger than (1). This completes the proof of the theorem.

\section{References}

1. R. P. Bambah and C. A. Rogers, Covering the plane with convex sets, J. London Math. Soc. 27 (1952), 304-314.

2. R. P. Bambah, C. A. Rogers, and H. Zassenhaus, On coverings with convex domains, Acta Arith. $9(1964), 191-207$.

3. C. H. Dowker, On minimum circumscribed polygons, Bull. Amer. Math. Soc. 50 (1944), 120-122.

4. L. Fejes Tóth, Über die dichteste Kreislagerung und dünnste Kreisüberdeckung, Comment. Math. Helv. 23 (1949), 342-349.

5. L. Fejes Tóth, Some packing and covering theorems, Acta Sci. Math. (Szeged) 12/A (1950), 62-67.

6. P. Gritzmann and J. M. Wills, On two finite covering problems of Bambah, Rogers, Woods, and Zassenhaus, Monatsh. Math. 99 (1985), 279-296.

7. C. A. Rogers, The closest packing of convex two-diemensional domains, Acta Math. 86 (1951), 309-321.

8. E. Sas, Über eine Extremaleigenschaft der Ellipsen, Compositio Math. 6 (1939), 468-470.

9. F. A. Valentine, Convex Sets, McGraw-Hill, New York, 1964.

Received September 20, 1985, and in revised form March 19, 1986. 